\title{
Conhecimento da enfermagem no tratamento de feridas
}

\author{
Knowledge of nursing in the treatment of wounds \\ Conocimiento de enfermería en el tratamiento de heridas
}

Cleuson Vieira Costa ${ }^{1 *}$, Elisete Silva Amorim², Francisca Franciana de Paiva ${ }^{3}$, Hadsan Taiana Aleixo de Fonseca ${ }^{4}$, Juniel Pereira Honorato ${ }^{4}$, Luiz Carlos Araújo de Souza ${ }^{5}$, Silvio Douglas Medeiros Costa ${ }^{4}$, Silvani Pereira dos Santos ${ }^{6}$, Simone Ribeiro Vieira ${ }^{6}$, Suenildo Messias da Silva ${ }^{7}$.

\section{RESUMO}

Objetivo: Buscar na literatura evidências quanto ao conhecimento dos enfermeiros no tratamento de feridas. Métodos: Trata-se de um estudo de revisão integrativa. Para obter as pesquisas relevante ao tema foi realizado a associação dos seguintes descritores: Enfermagem, feridas, tratamento, conhecimento, cuidados de enfermagem nas bases de dados Literatura Latino Americana e do Caribe em Ciências da Saúde (LILACS), Literatura Internacional em Ciências da Saúde (MEDLINE) que compõem a Biblioteca Virtual em Saúde (BVS). A amostra final foi composta por 10 artigos. Resultados: Todos ao estudo incluído na amostra estavam no idioma português (100\%), houve prevalência de estudo de campo (80\%) e a maioria das publicações foram concentradas no ano de 2020 (30\%). Com base na análise dos estudos pode-se evidenciar que as feridas são um problema atual no campo da saúde pública brasileira, tanto pela quantidade de pacientes que as desenvolvem quanto pela dificuldade que os profissionais da saúde enfrentam no cuidado com as mesmas. Considerações finais: $O$ presente estudo identificou baixo nível de conhecimento dos profissionais enfermeiros no tratamento de feridas.

Palavras-chave: Enfermagem, Feridas, Tratamento, Conhecimento, Cuidados de enfermagem.

\begin{abstract}
Objective: Search the literature for evidence regarding the knowledge of nurses in the treatment of wounds. Methods: This is an integrative review study. To obtain research relevant to the topic, the following descriptors were associated: Nursing, wounds, treatment, knowledge, nursing care in the databases Latin American and Caribbean Literature in Health Sciences (LILACS), International Literature in Health Sciences (MEDLINE) that make up the Virtual Health Library (VHL). The final sample consisted of 10 articles. Results: All of the study included in the sample were in Portuguese $(100 \%)$, there was a prevalence of field studies $(80 \%)$ and most publications were concentrated in the year $2020(30 \%)$. Based on the analysis of the studies, it can be seen that wounds are a current problem in the field of Brazilian public health, both because of the number of patients who develop them and because of the difficulty that health professionals face in caring for them. Final considerations: This study identified a low level of knowledge of professional nurses in the treatment of wounds.
\end{abstract}

Key words: Nursing, Wounds, Treatment, Knowledge, Nursing care.

\footnotetext{
${ }^{1}$ Faculdade Estácio de Macapá, Belém - PA. *E-mail: kleomcp65@hotmail.com

${ }^{2}$ Faculdade Metropolitana da Amazônia, Belém - PA.

${ }^{3}$ Faculdade Pan Amazônica, Belém - PA.

${ }^{4}$ Universidade da Amazônia (UNAMA), Belém - PA.

${ }^{5}$ Centro Universitário Metropolitano da Amazônia, Belém - PA.

${ }^{6}$ Centro Universitário de Lavras, Belém - PA.

${ }^{7}$ Faculdade Maurício de Nassau, Belém - PA.
} 


\section{RESUMEN}

Objetivo: Buscar en la literatura evidencia sobre el conocimiento del enfermero en el tratamiento de heridas. Métodos: Este es un estudio de revisión integradora. Para obtener la investigación relevante al tema, se asociaron los siguientes descriptores: Enfermería, heridas, tratamiento, conocimiento, cuidados de enfermería en las bases de datos Literatura Latinoamericana y del Caribe en Ciencias de la Salud (LILACS), Literatura Internacional en Ciencias de la Salud (MEDLINE) que hacen subir a la Biblioteca Virtual en Salud (BVS). La muestra final estuvo formada por 10 artículos. Resultados: Todo el estudio incluido en la muestra fue en portugués $(100 \%)$, hubo una prevalencia de estudios de campo $(80 \%)$ y la mayoría de las publicaciones se concentraron en el año 2020 (30\%). A partir del análisis de los estudios, se puede ver que las heridas son un problema actual en el campo de la salud pública brasileña, tanto por el número de pacientes que las desarrollan como por la dificultad que enfrentan los profesionales de la salud para atenderlas. Consideraciones finales: Este estudio identificó un bajo nivel de conocimiento del enfermero profesional en el tratamiento de heridas.

Palabras clave: Enfermería, Heridas, Tratamiento, Conocimiento, Atención de enfermería.

\section{INTRODUÇÃO}

A cicatrização de uma ferida é um processo sistemático e dinâmico, no qual a evolução da ferida será determinada pela implementação de cuidados de qualidade e específico para cada tipo de ferida, devendo ser levado em consideração fatores locais e sistêmicos, dentre eles a idade, doenças crônicas, uso de medicamentos, presença de infecção, edema, corpos estranhos, tecido necrótico, entre outros (MACHADO FS, et al., 2017).

O enfermeiro é o responsável por executar as etapas do tratamento de feridas, desta forma este pode realizar desde do acolhimento do paciente, avaliação da ferida, escolha do tratamento adequado até a cicatrização da ferida (SILVA PC, et al., 2021).

De acordo com a Resolução do Conselho Federal de Enfermagem 501/2015 é atribuição do enfermeiro o cuidado com feridas. Segundo a resolução o enfermeiro é autorizado a realizar consulta de enfermagem, prescrever e realizar curativo, coordenar e supervisionar a equipe de enf ermagem no tratamento e prevenção de feridas (BOTELHO LS, et al., 2020).

Segundo Júnior HG, et al. (2018) ao realizar a consulta de enfermagem a pessoas com feridas é necessário realiza a coleta de dados incluindo a investigação dos fatores intrínsecos e extrínsecos que podem prejudicar a cicatrização da ferida, bem como as características da lesão e a sua classificação quanto ao potencial de contaminação os sinais de inf ecção, características do leito e das bordas da ferida, a quantidade e aspecto do exsudato.

É importante que o enfermeiro também realize a orientação do paciente e esclareça todas as dúvidas sobre o tratamento e a importância da continuidade dos cuidados, para que este possa seguir suas orientações e tenha melhor adesão ao tratamento. De acordo com o autor é necessário que todas as informações sobre o paciente sejam documentadas com o intuito de contribuir na tomada de decisão terapêutica que determinara as intervenções mais favoráveis a cicatrização e auxiliara na avaliação dos resultados (MOTA EO, et al., 2017).

A assistência às pessoas com feridas pode ser realizada em diferentes ambientes, desde de ambientes hospitalares, serviços de Atenção Primária à Saúde ou clínicas especializadas, no entanto a assistência deve ter modelos sistematizados de cuidados dinâmicos que prezem pela segurança do cliente (SILVA GM, et al., 2017).

De acordo com Fonseca PMM, et al. (2019), o tratamento das feridas é realizado por métodos clínicos e cirúrgicos, sendo o curativo o tratamento clínico mais utilizado para realizar a reparação tecidual, desta forma é importante que o enfermeiro que trata de feridas tenha conhecimento vasto quanto aos mat eriais que serão 
utilizados no tratamento, a disponibilidade no mercado e também quanto a fisiologia da cicatrização, as suas etapas e ter a capacidade de adequar a assistência ao tratamento de cada uma delas, pois a fase inflamatória, proliferativa e reparadora determinará o avanço da cicatrização

Segundo Sousa MBV, et al. (2020), o tratamento de feridas inova cada vez mais com o desenvolvimento de novos produtos no mercado e as técnicas se aprimoram a cada dia, desta forma é importante que os profissionais, principalmente da enfermagem, realizem cursos de capacitação e atualização que direcionem e incremente nos planos de assistência de cada paciente.

Para Mota EO, et al. (2017), a educação em serviço na enfermagem é um método importante para o desenvolvimento e o aperfeiçoamento dos profissionais de enfermagem, contribuindo para uma assistência de qualidade, em que tanto o paciente quanto o profissional são beneficiados por meio de um processo produtivo ao educativo do ensino em serviço.

Diante disso o presente estudo teve o objetivo de buscar na literatura evidências quanto ao conhecimento dos enfermeiros no tratamento de feridas.

\section{MÉTODOS}

O estudo trata-se de uma revisão integrativa da literatura. O método permite a combinação de diversas metodologias (estudos experimentais e não-experimentais) e tem o potencial de desempenhar um papel importante na prática baseada na evidência em enfermagem (SOUSA LMM, et al., 2017).

Com o objetivo de def inir a amostra, houve o estabelecimento de critérios de inclusão e exclusão, a análise e discussão dos resultados. Com isso formulou-se a seguinte questão que orientou a elaboração do estudo: Quais evidências cientifica existe na literatura quanto o conhecimento dos enfermeiros no tratamento de feridas?

Para a elaboração do estudo foi realizado a busca de pesquisas nas seguintes bases de dados: Literatura Latino Americana e do Caribe em Ciências daSaúde (LILACS), Literatura Internacional em Ciências daSaúde (MEDLINE) que compõem a Biblioteca Virtual em Saúde (BVS). No intuito de obter estudo relevante ao tema foi realizado a associação dos seguintes descritores nas bases de dados: Enfermagem, feridas, tratamento, conhecimento, cuidados de enf ermagem.

Foram incluídos no estudo as pesquisas que estivessem disponíveis eletronicamente, na íntegra, no idioma português, inglês ou espanhol, e que foram publicados nos últimos 5 anos. Foi excluído da busca teses, capítulos de livros, artigos do tipo teórico-reflexivos, resumos, artigos duplicados nas bases de dados, textos incompletos eque não foram publicados nos últimos 5 anos. A pesquisa foi realizada no mês de Agosto de 2021.

Os estudos foram selecionados segundo os critérios de inclusão e exclusão no qual obteve-se 169 estudos como busca geral na base de dados LILACS, ao limitar a busca para texto completo e estudos que foram publicados nos últimos cinco anos obteve-se 28 estudos, destes foram analisados títulos e resultados onde apenas 6 estudos foi condizente com a questão desta pesquisa.

$\mathrm{Na}$ base MEDLINE, foi encontrado 45 estudos como busca total, ao realizar a filtragem dos estudos que limita as pesquisas em texto completo e que foram publicados nos anos de 2017, 2018, 2019, 2020 e 2021 obteve-se 15 estudos, destes foram analisados títulos e resultado no qual apenas 4 estudos foi condizente com a questão desta pesquisa.

$\mathrm{Na}$ fase final da busca dos estudos estes foram analisados quanto ao potencial de colaboração no estudo, avaliando o atendimento à questão norteadora da pesquisa, assim como os objetivos, amostra, método, desfechos, resultados e conclusão, resultando em 10 artigos.

Ao final 10 artigos atenderam a questão norteadora e forma adicionados ao estudo, como mostra a Figura 1.

REAEnf | Vol. 15 | DOI: https://doi.org/10.25248/REAEnf.e9221.2021 
Figura 1 - Fluxograma do processo de seleção dos estudos para a revisão integrativa.

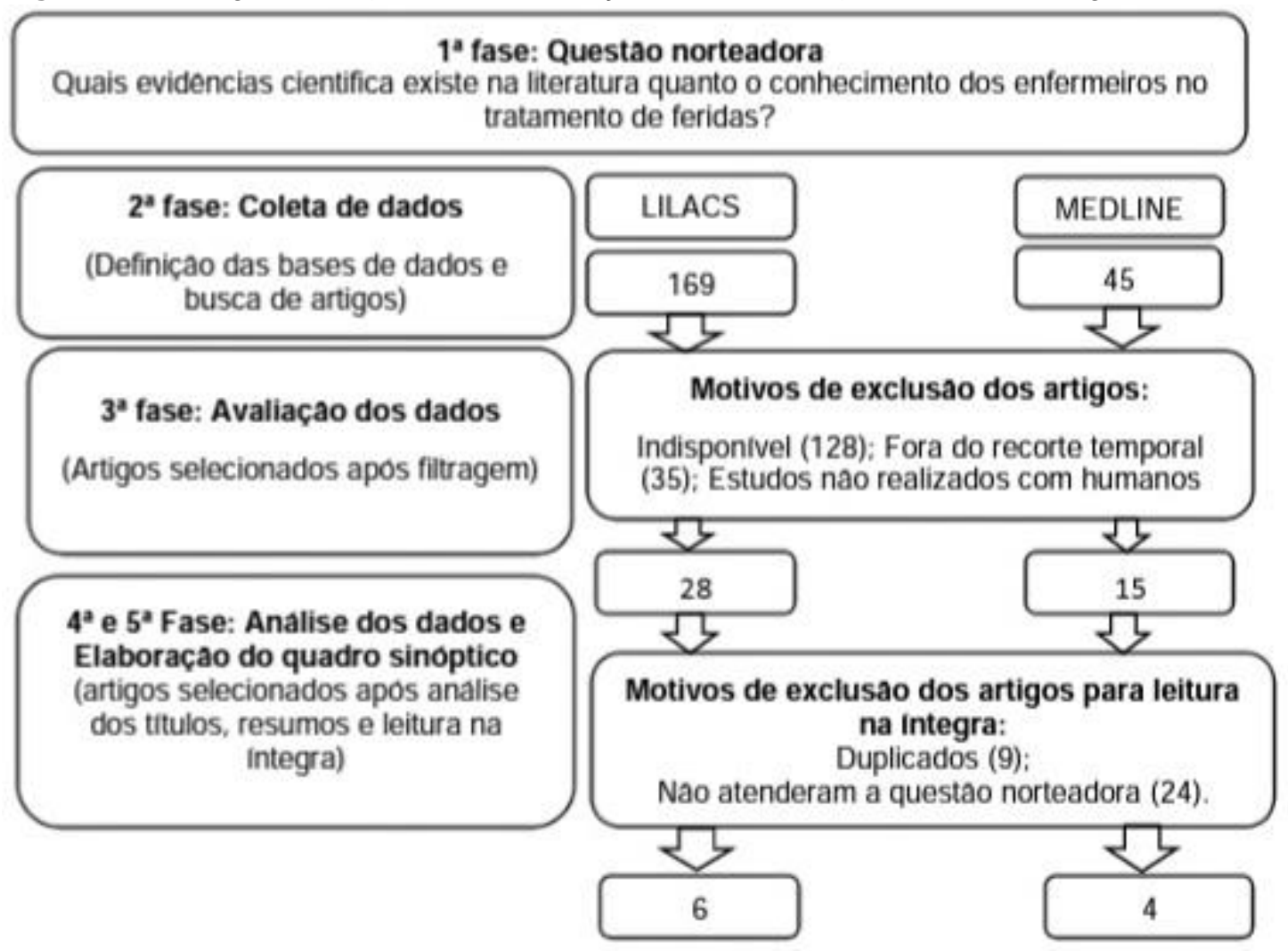

Fonte: Carvalho BL, et al., 2019.

\section{RESULTADOS E DISCUSSÃO}

A principal linha de pesquisa investigada no presente estudo versou sobre o conhecimento do enfermeiro quanto ao tratamento de feridas. Todos ao estudo incluído na amostra estavam no idioma português (100\%), houve prevalência de estudo de campo $(80 \%)$ e a maioria das publicações foram concentradas no ano de 2020 (30\%), como pode ser observado no Quadro 1.

Quadro 1 - Distribuição dos estudos segundo delineamento da pesquisa e ano de publicação, 2021.

\begin{tabular}{|c|c|c|}
\hline Autor & Delineamento da pesquisa & Ano \\
\hline BARBOSA A, et al. & Pesquisa de campo & 2020 \\
\hline COLARES CMP, et al. & Pesquisa de campo & 2021 \\
\hline DILL SM, et al. & Pesquisa de campo & 2018 \\
\hline LIMA BR, et a. & Pesquisa de campo & 2020 \\
\hline MITTAG BF, et a. & Pesquisa de campo & 2017 \\
\hline OLIVEIRA LSB, et al. & Pesquisa de campo & 2020 \\
\hline PAULA VAAL, et al. & Pesquisa de campo & 2019 \\
\hline RODRIGUES MELS, et al. & Revisão integrativa & 2021 \\
\hline SILVA ACX, et al. & Pesquisa de campo & 2019 \\
\hline SILVA BAB, et al. & Revisão integrativa & 2021 \\
\hline
\end{tabular}

Fonte: Carvalho BL, et al., 2019.

Ao analisar os estudos, de acordo com a temática abordada por cada um, pode-se observar que estes em sua maioria têm em comum o enfoque principal dado a importância do conhecimento do enfermeiro no tratamento de feridas. Desta forma os 10 estudos incluídos nesta revisão foram organizados segundo os autores e ano, amostra, objetivo e principais resultados (Quadro 2). 
Quadro 2 - Apresentação dos estudos segundo o autor, amostra, objetivo e principais resultados, 2021.

\begin{tabular}{|c|c|c|c|}
\hline Autor/ ano & Amostra & Objetivo & Principais resultados \\
\hline $\begin{array}{c}\text { BARBOSA A, } \\
\text { et al., } 2020\end{array}$ & $\begin{array}{c}33 \\
\text { enf ermeiros }\end{array}$ & $\begin{array}{l}\text { Avaliar o conhecimento } \\
\text { técnico-científico prestado } \\
\text { pelos enfermeiros aos } \\
\text { portadores de lesões } \\
\text { cutâneas. }\end{array}$ & $\begin{array}{l}\text { Os enfermeiros realizaram suas escolhas } \\
\text { com base no conhecimento técnico- } \\
\text { científico, pautados na etiologia das } \\
\text { feridas. }\end{array}$ \\
\hline $\begin{array}{l}\text { COLARES } \\
\text { CMP, et al., } \\
2021\end{array}$ & $\begin{array}{c}18 \\
\text { enfermeiros }\end{array}$ & $\begin{array}{c}\text { Determinar o nível de } \\
\text { conhecimento de enfermeiros } \\
\text { sobre cicatrização e } \\
\text { tratamento de feridas. }\end{array}$ & $\begin{array}{l}\text { Constatou-se baixo conhecimento nos } \\
\text { parâmetros: desbridamento, exsudato, } \\
\text { biofilme e sinais de infecção. }\end{array}$ \\
\hline $\begin{array}{l}\text { DILL SM, et } \\
\text { al., } 2018\end{array}$ & $\begin{array}{c}21 \\
\text { enfermeiros }\end{array}$ & $\begin{array}{l}\text { Avaliar o conhecimento } \\
\text { técnico-científico dos } \\
\text { enfermeiros relacionado ao } \\
\text { tratamento de feridas. }\end{array}$ & $\begin{array}{c}\text { Há necessidade de capacitar os } \\
\text { enfermeiros da instituição quanto aos } \\
\text { aspectos relacionados à avaliação do } \\
\text { paciente, da ferida e tipos de coberturas } \\
\text { Disponíveis. }\end{array}$ \\
\hline $\begin{array}{l}\text { LIMA BR, et } \\
\text { al., } 2020\end{array}$ & 12 pacientes & $\begin{array}{l}\text { Analisar as prescrições de } \\
\text { curativo realizadas por } \\
\text { enfermeiros e médicos. }\end{array}$ & $\begin{array}{c}\text { Cerca de } 93,9 \% \text { prescrições estavam } \\
\text { incompletas, sendo que em } 31,4 \% \text { foi } \\
\text { encontrado divergência entre prescrições } \\
\text { de enfermagem e medica no mesmo } \\
\text { registro diário. }\end{array}$ \\
\hline $\begin{array}{l}\text { MITTAG BF, } \\
\text { et al., } 2017\end{array}$ & $\begin{array}{c}25 \\
\text { enf ermeiros }\end{array}$ & $\begin{array}{l}\text { Identificar atividades de } \\
\text { Enfermagem relacionadas às } \\
\text { lesões de pele. }\end{array}$ & $\begin{array}{l}\text { A mudança de decúbito do paciente é a } \\
\text { medida de prevenção mais utilizada em } \\
\text { úlceras por pressão, no tratamento é a } \\
\text { realização de curativos. No estudo } 88 \% \\
\text { declarou que não existe política educativa } \\
\text { sobre o cuidado com a pele. }\end{array}$ \\
\hline $\begin{array}{l}\text { OLIVEIRA } \\
\text { LSB, et al., } \\
2020\end{array}$ & $\begin{array}{c}22 \\
\text { enf ermeiros } \\
\text { e } 15 \\
\text { auxiliares } \\
\end{array}$ & $\begin{array}{l}\text { Avaliar o conhecimento da } \\
\text { equipe de enfermagem com } \\
\text { relação ao tratamento de } \\
\text { feridas. }\end{array}$ & $\begin{array}{l}\text { Identificou baixo conhecimento nos } \\
\text { parâmetros: desbridamento, exsudato, } \\
\text { biofilme e sinais de infecção. }\end{array}$ \\
\hline $\begin{array}{l}\text { PAULA } \\
\text { VAAL, et al., } \\
2019\end{array}$ & $\begin{array}{c}32 \\
\text { enfermeiros }\end{array}$ & $\begin{array}{l}\text { Caracterizar o perfil da } \\
\text { formação e atualização dos } \\
\text { enf ermeiros assistenciais e } \\
\text { avaliar o conhecimento sobre } \\
\text { o tratamento de feridas. }\end{array}$ & $\begin{array}{l}\text { O conhecimento geral dos entrevistados } \\
\text { foi considerado bom. Cerca de } 78 \% \text { dos } \\
\text { profissionais informaram se atualizar } \\
\text { sobre os cuidados com feridas. }\end{array}$ \\
\hline $\begin{array}{l}\text { RODRIGUES } \\
\text { MELS, et al., } \\
\quad 2021\end{array}$ & 11 artigos & $\begin{array}{l}\text { Identificar a importância da } \\
\text { atuação em conjunto da } \\
\text { equipe enfermagem nos } \\
\text { cuidados das feridas. }\end{array}$ & $\begin{array}{l}\text { O enfermeiro tem autonomia no cuidado } \\
\text { e deve manter-se atualizado, além de } \\
\text { transmitir para sua equipe, o } \\
\text { direcionamento para as estratégias } \\
\text { voltadas à construção do protocolo do } \\
\text { cuidado com feridas. }\end{array}$ \\
\hline $\begin{array}{l}\text { SILVA ACX, } \\
\text { et al., } 2019\end{array}$ & $\begin{array}{c}35 \\
\text { enf ermeiros }\end{array}$ & $\begin{array}{l}\text { Analisar o conhecimento do } \\
\text { enfermeiro sobre a Terapia } \\
\text { por Pressão Negativa (TPN) } \\
\text { como influência no } \\
\text { tratamento do paciente com } \\
\text { ferida complexa. }\end{array}$ & $\begin{array}{c}\text { Observou-se uma limitação do } \\
\text { conhecimento dos enfermeiros como } \\
\text { influência no tratamento } \\
\text { do paciente em uso da TPN, visto a } \\
\text { razoável assistência prestada com essa } \\
\text { modalidade de curativo. }\end{array}$ \\
\hline $\begin{array}{l}\text { SILVA BAB, } \\
\text { et al., } 2021\end{array}$ & 18 artigos & $\begin{array}{c}\text { Analisar as evidências } \\
\text { científicas sobre a } \\
\text { contribuição dos cursos de } \\
\text { capacitação em feridas para } \\
\text { a realização de curativos pela } \\
\text { equipe de enfermagem. }\end{array}$ & $\begin{array}{c}\text { Após a realização das intervenções } \\
\text { educativas os enfermeiros sentiram-se } \\
\text { mais preparados para avaliar e realizar } \\
\text { tratamento e acompanhamento de } \\
\text { feridas. }\end{array}$ \\
\hline
\end{tabular}

Fonte: Carvalho BL, et al., 2019.

Com base na análise dos estudos pode-se evidenciar que as feridas são um problema atual no campo da saúde pública brasileira, tanto pela quantidade de pacientes que as desenvolvem quanto pela dificuldade que 
os profissionais da saúde enf rentam no cuidado com as mesmas, para que haja uma boa evolução e resultado satisfatório no final do tratamento (SILVA ACX, et al., 2019)

O tratamento de feridas sempre foi uma atividade cotidiana na prática da enfermagem, sendo está prática reconhecida como uma competência essencial do enfermeiro. Desta forma é de suma importância que estes profissionais tenham conhecimento teórico baseado em evidências que garantam a qualidade da assistência ao paciente com feridas (MITTAG BF, et al., 2017).

De acordo com Rodrigues MELS, et al. (2021), o enfermeiro é o profissional responsável por avaliar a lesão, muitas das vezes determinar o tipo de tratamento que será aplicado, orientar e supervisionar a realização dos curativos pela equipe de enfermagem. Segundo o autor a escolha do trat amento bem como do curativo, técnica e cobertura deve facilitar a cicatrização, porém se mal escolhidos pode não somente retardar sua cicatrização como também agravar sua condição ainda mais.

Estudo realizado por Dill SM, et al. (2018), que buscou avaliar o conhecimento de 21 enfermeiros quanto ao tratamento de feridas, identificou a necessidade de capacitar os enfermeiros da instituição na qual foi realizada a pesquisa quanto aos aspectos relacionados à avaliação do paciente, da ferida e os tipos de coberturas disponíveis.

Na pesquisa $52,38 \%$ dos profissionais af irmaram que a conduta do tratamento das feridas é prescrita pelo médico, fato este que demonstra a falta de autonomia dos enfermeiros, $72,22 \%$ dos enfermeiros não abordaram os aspectos mais relevantes sobre a avaliação do paciente (DILL SM, et al., 2018).

Cerca de $52,38 \%$ não descreveram de forma correta os aspectos da ferida, $38,10 \%$ indicaram o PVPI para limpeza do leito da ferida, fato este preocupante pois o uso de PVPI é contraindicado para essa finalidade por possui ação citotóxica para os fibroblastos (DILL SM, et al., 2018).

Resultado similar foi observado no estudo realizado por Colares CMP, et al. (2019), com 22 enfermeiros que constatou baixo conhecimento dos profissionais com relação ao desbridamento, biofilme, exsudato e os sinais de infecção. O autor identificou em seu estudo que $48,5 \%$ dos produtos foram indicados de forma inadequada pelos profissionais, assim como o tempo de permanência (92,9\%) dos produtos com recomendação de troca entre cinco e sete dias.

Santana AC, et al. (2019), evidenciou em seu estudo fragilidade no processo de atendimento da enfermagem ao tratar pessoas com ulceras vasculares. No estudo o autor identificou que dos 218 profissionais que participaram da pesquisa $58,3 \%$ nunca realizaram atualizações em avaliação e tratamento de feridas, $40,7 \%$ dos profissionais revelaram pouca experiência na área. Os dados são preocupantes visto que cerca de $10 \%$ desses enfermeiros atuam de modo fixo nas salas de curativos e os demais participam do atendimento quando chamados.

Corrobora com o achado o estudo realizado por Silva BAB, et al. (2021), que identificou lacunas no conhecimento dos profissionais quanto ao processo de cicatrização, características da lesão, indicação e troca de cobertura. O autor ressalta a importância de intervenções educativas como estratégias eficazes para a atualização do conhecimento da equipe de enfermagem.

Oliveira LSB, et al. (2020), também destaca em seu estudo as intervenções educativas aos profissionais de enfermagem com relação ao tratamento de feridas, ao avaliar o conhecimento de 22 enfermeiros e 15 auxiliares de enfermagem em dois hospitais públicos no estado do Piaú, antes e depois de uma atividade de capacitação profissional.

O resultado do estudo evidenciou que apenas $11 \%$ dos profissionais tinham especialização em feridas, e que antes da atividade de capacitação o questionamento relacionado ao tratamento de feridas foi respondido corretamente por apenas $67,5 \%$ dos participantes, e que após a intervenção da capacitação o mesmo questionamento foi respondido corretamente por 90,8\% dos participantes (OLIVEIRA LSB, et al., 2020).

Corrobora com o achado o estudo realizado por Lima BR, et al. (2020), que identificou em sua pesquisa fragilidades nas prescrições de curativo. Cerca de 93,9\% das prescrições estavam incompletas, sendo que em $31,4 \%$ foi encontrado divergência entre prescrições de enfermagem e medica no mesmo registro diário.

REAEnf | Vol. 15 | DOI: https://doi.org/10.25248/REAEnf.e9221.2021 
Observou-se ainda que não havia especificação do produto a ser utilizado no curativo em $32,3 \%$ e que a prescrição do produto foi inadequada as características das feridas em $38,3 \%$. Para o autor o resultado da pesquisa reflete a dificuldade da equipe multiprofissional em articular de forma conjunta as condutas terapêuticas a serem adotadas, assim como a falta de capacitação profissional com relação ao tratamento de feridas (LIMA BR, et al., 2020).

No estudo de Ferreira AM (2018), os enfermeiros obtiveram, em média $69,4 \%$ de acertos quando lhes questionados a respeito do cuidado de pacientes com feridas, das 12 questões sobre o conhecimento do cuidado aos pacientes com feridas, somente 6 tiveram acertos acima de $70 \%$ e 3 acima de $90 \%$, apenas uma das 12 questões foi respondida corretamente por todos os enfermeiros. Na pesquisa os enfermeiros apresentaram conhecimento insuficiente em algumas áreas referentes ao tema, tais como a utilização da Escala de Braden para avaliação do risco e a identificação dos sinais e sintomas clássicos de infecção. Segundo o autor é necessário a atualização destes profissionais quanto aos avanços das evidências atuais que embasam o cuidado aos pacientes com feridas.

Diverge dos resultados acima a pesquisa realizada por Paula VAAI, et al. (2019), em um hospital público de ensino da Zona da Mata Mineira, com 32 enfermeiros. No estudo a autora identificou que $78 \%$ dos profissionais realizaram atualizações sobre os cuidados com feridas, $43,8 \%$ dos profissionais afirmaram que o enfermeiro era o responsável por avalia a ferida e prescreve o tipo de curativo adequado para o tratamento, fato este que evidência a autonomia do enfermeiro no cuidado com feridas.

Resultado similar foi observado no estudo de Barbosa A, et al. (2020), que também mostrou resultados satisfatórios quanto ao nível de conhecimento dos enfermeiros no tratamento de feridas. No estudo 21 enfermeiros possuíam pós-graduação, 2 profissionais eram especializados em estomaterapia, 12 dos entrevistados apresentavam mais de 10 anos de atuação na área de feridas.

Cerca de 10 enfermeiros participaram de capacitações e atualização relacionadas a tratamento de feridas há menos de 6 meses. Para o autor o conhecimento dos enfermeiros pesquisados revelou-se extremamente relevante tanto para o manejo das feridas, quanto para garantir a qualidade da assistência prestada (BARBOSA A, et al., 2020).

No entanto pode-se identificar ausência de protocolos que padronize a assistência aos pacientes com feridas na instituição de pesquisa, desta forma os profissionais realizaram suas escolhas com base no conhecimento técnico-científico, pautados na etiologia da lesão, nas características de cada paciente e nos produtos disponíveis na instituição. Corrobora com o achado o estudo de Paula VAAI, et al. (2019), no qual $68,8 \%$ dos profissionais informaram não existir ou não saber da existência de protocolo de prevenção ou tratamento de feridas na instituição.

De acordo com Rezende GS, et al. (2021) os protocolos têm a finalidade de organizar a assistência de enfermagem no tratamento de feridas e contribuir para a diminuir os custos em saúde. Respaldando as condutas de avaliação, diagnóstico, planejamento, cuidado, tratamento, evolução e registro dos dados dos usuários, contribuindo para autonomia dos profissionais, favorecendo os resultados ao tratamento dos pacientes e diminuindo os custos para as instituições.

Segundo Paula VAAL, et al. (2019), a existência de um protocolo assistencial interfere na qualidade da assistência por ser uma ferramenta sistematizada que facilita a avaliação do tratamento e descreve os passos para a realização de acordo com a realidade da instituição. Rezende GS, et al. (2021) relata em seu estudo que os protocolos quando utilizados no cuidado as feridas resultam em benefícios para o paciente e cria oportunidades para futuras intervenções, pois estes servem para orientar os profissionais a obter informações sobre o estado de saúde do paciente e características que proporcionará ao mesmo um tratamento eficaz e uma reabilitação completa.

\section{CONSIDERAÇÕES FINAIS}

O presente estudo identificou baixo nível de conhecimento dos profissionais enfermeiros no tratamento de feridas, observou-se que a deficiência do conhecimento está desde a avaliação do paciente até a escolha do 
tratamento adequado para cada tipo de ferida. Desta forma ressalta-se a necessidade de capacitação dos profissionais assim como a elaboração de protocolos que sistematize o cuidado em feridas, visto que a enfermagem tem autonomia para tratar feridas tanto em hospitais como em clinicas e unidades básicas de saúde. A elaboração do presente estudo é importante por colaborar para construção do conhecimento teórico dos profissionais da enfermagem.

\section{REFERÊNCIAS}

1. BARBOSA A, et al. Percepção dos enfermeiros na terapia de feridas: Tratamento e coberturas. Revista Feridas, 2020; 8(40): 1400-1400.

2. BOTELHO LS, et al. Atuação do enfermeiro no cuidado a prevenção e tratamento de lesões por pressão. Research, Society and Development, 2020;9(7):569-573.

3. CARVALHO BL, et al. Assistência de enfermagem a pacientes com estoma intestinal. Revista Eletrônica Acervo Saúde, 2019;24(604):01-08.

4. COLARES CMP, et al. Cicatrização e tratamento de feridas: a interface do conhecimento à prática do enfermeiro. Enfermagem em Foco, 2019;10(3): 52-58.

5. DILL SM, et al. Avaliação do conhecimento dos enfermeiros de uma fundação de saúde comunitária do município de Sinop/MT sobre o tratamento de feridas. Scientific Electronic Archives, 2018;11(2):569-575.

6. FERREIRA AM, et al. Conhecimento e prática de enfermeiros sobre cuidados aos pacientes com feridas. J. res.: fundam. care. Online, 2013;6(3):1178-1190.

7. FONSECA PMM, et al. A atuação da equipe de enfermagem frente aos cuidados do paciente portador de ferida venosa. Enferm. Foco, 2019;4(1): 67-72.

8. JÚNIOR HG, et al. Processo de enfermagem na assistência a pacientes com feridas em cicatrização por segunda intenção. Cogitare Enferm, 2018;4(23):56-65.

9. LIMA BR, et al. Avaliação das prescrições de enfermagem e médicas para curativos em um hospital universitánio: Implicações éticas. Enferm. Foco 2020;11(5): 67-72.

10. MACHADO FS, et al. Perspectiva do enfermeiro frente à assistência no tratamento de feridas em ambiente hospitalar. R Epidemiol Control Infec, 2017;7(3):134-139.

11. MITTAG BF, et al. Cuidados com Lesão de Pele: Ações da Enfermagem. rev. Estima, 2017;15(1):19-25.

12. MOTA EO, et a. Aplicação da técnica de curativo pela equipe de enfermagem em uma unidade de pronto atendimento. International Nursing Congress, 2017;9(12):59-68.

13. OLIVEIRA LSB, et al. Os efeitos da capacitação da equipe de enfermagem sobre avaliação e cuidado de pacientes com feridas. Braz. J. of Develop. 2020;6(5): 29707-29725.

14. PAULA VAAI, et al. O conhecimento dos enfermeiros assistenciais no tratamen to de feridas. rev. Enferm. Foco, 2019; 45(1): 13-21.

15. REZENDE GS, et al. Protagonismo do enfermeiro no processo de cicatrização das feridas crônicas: Um ensaio da literatura. revista científica multidisciplinar, 2021;2(4):68-75.

16. RODRIGUES MELS, et al. Importância da atuação de enfermagem nos cuidados das feridas. Revista inter saúde. 2021;1(4): 768-773.

17. SANTANA AC, et a. Caracterização de profissionais de enfermagem que atendem pessoas com úlceras vasculares na rede ambulatorial. Rev Bras Enferm,2013;66(6):821-6.

18. SILVA GM, et al. A importância da avaliação multidisciplinar no tratamento de feridas crônicas. International nursing congress, $2017 ; 9(12): 86-91$.

19. SILVA PC, et al. A atuação do enfermeiro no tratamento de feridas. Brazilian Journal of Health Review, 2021; 4(2): 4815-4822.

20. SILVA ACX, et al. Terapia por pressão negativa (TPN): O conhecimento do enfermeiro como influência no tratamento do paciente com ferida complexa em uma instituição hospitalar de Belo. Horizonte/MG. Revista Feridas, 2019;07(38): 1369-1376.

21. SILVA BAB, et al. Capacitação em feridas para a realização de curativos pela equipe de enfermagem: Revisão integrativa. Rev Enferm Atual In Derme, 2021;95(34):821-828.

22. SOUSA MBV, et al. Assistência de enfermagem no cuidado de feridas na atenção primária em saúde: revisão integrativa. Revista Eletrônica Acervo Saúde, 2020; 48(9):333-338.

23. SOUSA LMM, et al. A metodologia de revisão integrativa da literatura em enfermagem. Revista investigação em enfermagem, 2017;17(2):56-60. 